\title{
Gut Microbiota Profiles in Early- and \\ Late-Onset Colorectal Cancer: A Potential Diagnostic Biomarker in the Future
}

\author{
Murdani Abdullah ${ }^{a, b}$ Ninik Sukartini ${ }^{c}$ Saskia Aziza Nursyirwan ${ }^{a}$ \\ Rabbinu Rangga Pribadi ${ }^{a}$ Hasan Maulahela ${ }^{a}$ Amanda Pitarini Utari ${ }^{a}$ \\ Virly Nanda Muzellina ${ }^{a}$ Agustinus Wiraatmadja ${ }^{a}$ Kaka Renaldi ${ }^{a}$
}

${ }^{a}$ Division of Gastroenterology, Department of Internal Medicine, Faculty of Medicine, University of Indonesia, Dr. Cipto Mangunkusumo National General Hospital, Jakarta, Indonesia; bHuman Cancer Research Center, Indonesian Medical Education and Research Institute, Faculty of Medicine, University of Indonesia, Jakarta, Indonesia;

'Department of Clinical Pathology, Faculty of Medicine, University of Indonesia, Dr. Cipto Mangunkusumo National General Hospital, Jakarta, Indonesia

\section{Keywords}

Colorectal cancer - Early-onset colorectal cancer · Gut microbiota $\cdot$ Microbiota dysbiosis · Diagnostic biomarker

\begin{abstract}
Background: Researchers believe the role of gut microbiota dysbiosis in the raised incidence of early-onset colorectal cancer (EOCRC). The development of EOCRC may be associated with microbiota dysbiosis either dependently or independently (combined with other risk factors). Summary: Recently, the rising of incidence and mortality of EOCRC have been noted. Some researchers are looking for risk factors influencing this fact. They hypothesize that it may be because of microbiota dysbiosis. Microbiota dysbiosis has been known to promote cancer development through immunity dysregulation and chronic inflammation. Microbiomes profile in late-onset colorectal cancer (LOCRC) among older patients has been documented, but there is still lack of data about microbial profiles among younger colorectal cancer (CRC) patients. This review tries to explain microbial profiles differences between EOCRC and LOCRC as a potential diagnostic biomarker in the future, and whether microbiota can have a role in EOCRC genesis. Key Messages: Mi-
\end{abstract}

karger@karger.com

(c) 2021 S. Karger AG, Basel

www.karger.com/dig

Karger" crobiota does vary with age, and EOCRC may be associated with colonization of some specific bacteria. Further studies about gut microbiota profiles in EOCRC and LOCRC may provide a new insight on diagnostic biomarker of CRC.

(c) 2021 S. Karger AG, Basel

\section{Introduction}

Based on GLOBOCAN 2018 data, colorectal cancer (CRC) is the third most lethal and the fourth most commonly diagnosed cancer all over the world for both sexes [1]. CRC incidence has been rising especially in the developing countries worldwide [1-3]. CRC is the second most common cause of death in US [4]. CRC has caused about 500,000 deaths per year worldwide [5]. Around 5\% of the population in Western countries will develop CRC [6].

CRC is widely considered as a disease that affects people after fifth decade of life (late-onset CRC [LOCRC]), but recently a greater incidence of young-age CRC (earlyonset CRC [EOCRC]) patients have been documented [3, 7]. In some literatures, EOCRC is defined as patients who are diagnosed with $\mathrm{CRC}<50$ years of age $[8,9]$. American Cancer Society in 2020 reported declines in incidence and 
mortality of CRC patients older than 65 years $(3.3$ and 3\% annually). Interestingly, among patients younger than 50 years, the incidence and mortality rate have increased (2 and $1.3 \%$ annually) [4]. In Dr. Cipto Mangunkusumo National General Hospital, Indonesia, the incidence of CRC $<30$ years old increased from $4.4 \%$ in $2002-2006$ to $9 \%$ in 2007-2011 ( $p=0.051)$ [10]. Among 316 CRC patients during 2014-2016 in the same hospital, there were 38 patients aged $<45$ years $(26.8 \%)$ [11]. This increase in EOCRC is believed by researchers because of a more sedentary lifestyle, modern dietary factors, and obesity $[3,8,12]$.

Nowadays, the study of intestinal microbiota has provided a new biomarker for CRC because many metabolic, structural, and immunological functions are performed by microbiota [13]. Gut microbiota dysbiosis (imbalance of normal content) and chemical compounds produced by microbiota (acetaldehyde and N-nitrates) are key factors for chronic inflammation $[6,14]$. Gut microbiota can induce CRC development by inducing chronic inflammation, producing toxic metabolites, and changing stem cell dynamics [15]. The inflammation induces environmental changes and colonization of other bacteria which promotes carcinogenesis [14].

EOCRC incidence has risen and some scientists have hypothesized the contribution of intratumoral microbiota because there were not any significant genetic differences between younger and older CRC patients. Certain microbes can induce inflammation and disturb the lining of the colon. These can promote DNA damage and mutation which then lead to cancer [16]. This review will discuss about EOCRC, role of gut microbiota in CRC pathogenesis, and microbial profiles in CRC patients. We will finally discuss about the difference between gut microbiota profiles in EOCRC and LOCRC as a potential biomarker in the future, which is still an interesting topic to be studied nowadays.

\section{CRC and EOCRC}

Accumulation of genetic and environmental factors influences CRC genesis. Most of CRC occurs sporadically and less than one fifth of CRC are hereditary. Some risk factors are alcohol, smoking, and obesity. Consuming a lot of red meat, fat, and processed food, accompanied by low intake of fibers also have a role in CRC pathogenesis [1-3].

Genetic predisposition and environmental condition contribute to an increase in the incidence of CRC in young adult population. Prevention and early detection are very important precautions to be taken in symptom- atic young adults $[4,5]$. American Cancer Society recommends that 45 years old or older adults with an average risk of CRC have to be screened with the stool test or visual examination. All positive results in noncolonoscopy test must be continued with colonoscopy procedures [6].

Of young-age CRC, 15-20\% are hereditary, so both patients and doctors should be aware about the importance of a family genogram [7, 8]. A study from 2011 to 2017 found that compared with LOCRC, EOCRC patients tended to have inflammatory bowel disease (IBD) $(\mathrm{OR}=2.97 ; 95 \% \mathrm{CI}, 1.16-6.63)$ and CRC family history $(\mathrm{OR}=2.87 ; 95 \% \mathrm{CI}, 1.89-4.25)$ [9]. Symptomatic young adults should be endoscopically investigated to avoid a late diagnosis [7]. About $>80 \%$ of the young-onset CRCs are symptomatic at the time of diagnosis: $51 \%$ rectal bleeding, 32\% abdominal pain, $18 \%$ change in bowel habits, and $13 \%$ weight loss [10]. The incidence of young-age CRC has increased mainly between 40 and 49 years of age. They are more likely to be found in the distal colon-rectum (left-sided CRC) and at the advanced stages $[7,11]$. Keshinro et al. [12] reported that EOCRC was more probable to be presented in the left colon than LOCRC ( 81 and $45 \%, p=0.001)$. Gausman et al. [9] also showed that EOCRC was more likely to be presented in the left colon or rectum (75 and 59\%, $p=0.02)$ and at a later stage than LOCRC (77 and 62\%, $p=0.01$ ).

Some screening strategies have been established to reduce the mortality of CRC patients; such as the blood and stool test, tissue sample, radiographic examination, endoscopy, and computed tomography colonography. The fecal occult blood test and fecal immunochemical test taken nowadays have been initial and noninvasive tests for CRC, although have moderate sensitivity and specificity $[6,13-15]$. Fecal sample is easier and less invasive than colonic mucosa tissue sample from biopsy. Although it is more invasive than fecal sample, mucosal biopsy can provide a better view on microbiota for CRC diagnosis and prognosis [1]. Tumor mucosal samples are often compared with normal mucosa in evaluating the microbiota. Some studies showed that there were differences between fecal and mucosal microbiota profiles [16]. Flemer et al. [17] in 2017 studied that fecal microbiota did not represent total CRC gut microbiota and also there was a microbiota population difference between proximal and distal cancer. Colonoscopy also has limitations. Nonpolypoid flat lesions especially in the right colon may be missed [18]. Other considerations are bowel preparation and patients' fear of pain and anxiety [19]. Currently, colonic microbiota has begun to be studied as a potential diagnostic tool for CRC. 


\section{Gut Microbiota and Colorectal Carcinogenesis}

The colon has a diverse microbial ecosystem which facilitates the host immune system. Microbial populations consist of mainly bacteria, viruses, archaea, eukaryotes, and fungi [13]. They contain at least 100 trillion bacteria with around $1.5 \mathrm{~kg}$ biomass $[20,21]$. The gut microbiota plays an important role in nutritional absorption, immunity, tissue repair, and also carcinogenesis [1]. Intestinal epithelial cells form a physical barrier and are continuously replenished by multipotent stem cells [22]. Communications between host cells and microbiota facilitate homeostatic and structural integrity. In homeostatic conditions, intestinal microbiota such as Roseburia intestinalis and Faecalibacterium prausnitzii ferment complex carbohydrates into short-chain fatty acids (SCFAs) [23]. SCFAs can regulate host immune response by binding with G-protein coupled receptors, and then suppress local inflammation, and maintain intestinal layer coherence [24].

This interaction and microbiota composition become maladapted in CRC, called dysbiosis. Bacteria can produce toxin then disrupt epithelial barrier defense and induce inflammation, DNA damage, and cancer growth [25]. After that, mesenchymal cells act as a second line of defense by starting signal networks to maintain epithelial integrity such as activation of NF- $\kappa \mathrm{B}$ and STAT3 pathways for tissue repair $[26,27]$. In dysbiosis condition, Enterobacteriaceae (such as Salmonella and Shigella) induce neutrophil transepithelial migration then reduce the number of SCFAs-producing bacteria and promote the growth of inflammatory pathogenic bacteria $[24,28]$.

TNF- $\alpha$, IL- 6 , NF- $\kappa B$, and reactive oxygen species have a role in cell proliferation and DNA damage; therefore, contribute to carcinogenesis [29]. Reactive oxygen species produced by neutrophils, macrophages, and natural killer cells can increase the cyclooxygenase-2 (COX-2) level which plays role in chronic IBD [30]. A 15\% increased risk of developing CRC is well-found among IBD patients [31]. Toll-like receptors, a part of pattern recognition receptors, can recognize gut barrier disruption by microbiomes then promote protective immunity by inducing cytokines expression [20,32]. Toll-like receptors are responsible to coordinate microbiota population and maintain mucosal integrity which is started during neonatal life [33].

A driver-passenger theory by Tjalsma et al. [34] had been accepted to explain the role of microbiota in carcinogenesis. Some local bacteria, named as "driver bacteria," initiate cancer by triggering DNA damage. For example, colibactin genotoxin produced by Escherichia coli

Gut Microbiota Profiles in Early- and Late-Onset CRC can promote DNA damage and induce cell mutation [35, 36]. Fragilysin by $B$. fragilis also can stimulate DNA damage and promote degradation of E-cadherin, a tumor suppressor protein, which then causes cell multiplication [37]. Fearon proposed a CRC mutational sequence. It starts with the adenomatous polyposis coli gene mutation, which causes transition to adenoma, then continues with P53 mutation which leads to carcinoma [38].

In the next step, rupturing and bleeding of cancer tissues accompanied by ongoing carcinogenesis may alter the surrounding microenvironment and promote the growth of other bacteria, called "passenger bacteria." [34]. During the first bacterial invasion, these bacteria do not play any roles [39]. These bacteria include tumor opportunistic pathogens (Fusobacterium and Streptococcus gallolyticus) and probiotics (family Coriobactericeae, genus Roseburia and Faecalibacterium) [40]. Fusobacterium nucleatum can induce pro-inflammatory cytokines and suppress T-cell activity, associated with IBD and CRC development [41]. Coriobacteriaceae are considered as probiotics because they convert dietary parts into useful metabolites such as antioxidants and butyrate, which give energy to colonocytes [42].

In summary, gut dysbiosis induces "driver bacteria" colonization, which promotes chronic inflammation. New "passenger bacteria" are introduced by the inflammation because of microenvironmental changes. After that, the process continues to adenomatous then tumor formation [1].

Other theory named "keystone pathogen (or a-bug)" was proposed by Hajishengallis et al. [43] Keystone pathogens are small amount bacteria which have oncogenic properties and can remodel microbial composition, mucosal integrity, and immune response [21, 43]. Small amount of enterotoxigenic B. fragilis (ETBF), E. coli, and Fusobacterium can induce dysbiosis, alter colonic integrity, cause inflammation with their toxins, and promote tumorigenesis [44].

CRC carcinogenesis in general can be split into 3 stages: initiation, promotion, and progression, shown in Figure 1 . The initiation stage is defined by DNA damage induced by specific carcinogenic agent which then promote a proliferative advantage $[45,46]$. Polyketide synthase gene cluster $E$. coli, which can produce colibactin toxin is an example of the initiation stage [47]. The second stage, promotion, consists a proliferation of preneoplastic cells, more induction to DNA damage, and cellular mutation $[45,46]$. Fragilysin produced by $B$. fragilis can disrupt colonic integrity then induce procarcinogenic T-helper 17 cells [48]. The final stage, progression, is marked by fur- 


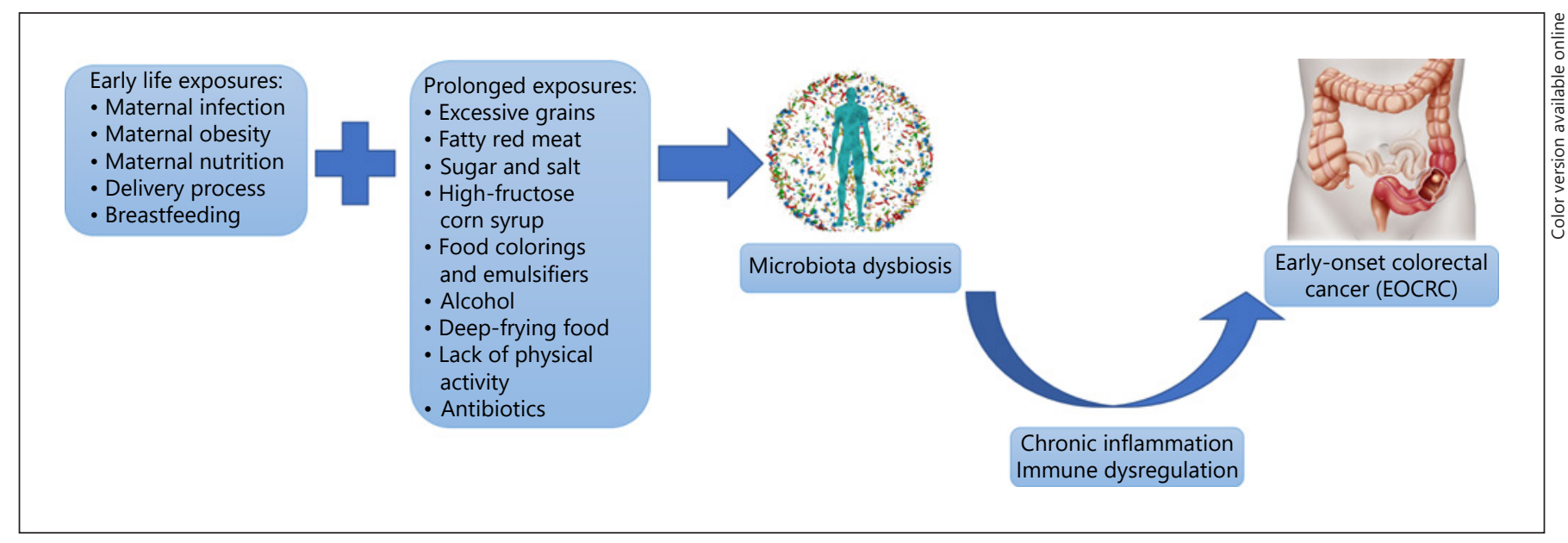

Fig. 1. Colorectal carcinogenesis stages. There are 3 stages of colorectal carcinogenesis. Each stage is influenced by specific bacteria and their products $[45,46]$.

Fig. 2. EOCRC pathogenesis. Early-life exposures from pregnancy, delivery until neonatal life are combined with prolonged exposures during childhood and adolescence. These factors affect intestinal microbiota, leading them to dysbiosis condition. Prolonged microbiota dysbiosis then leads to chronic inflammation and immune system dysregulation which are responsible for EOCRC development [50]. EOCRC, early-onset colorectal cancer.

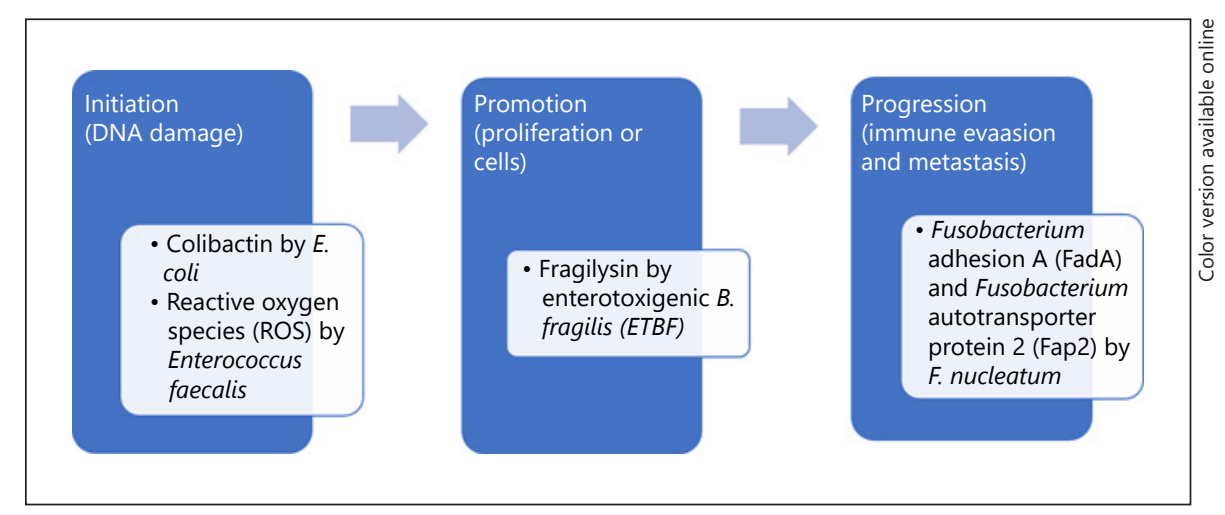

ther tumor growth, invasion, and metastases [46]. Fusobacterium binds to E-cadherin using FadA adhesins to promote more proliferation and invasion [49].

Folate and biotin produced by microbiota are responsible for epithelial proliferation and cancer afterward [50]. Clostridium produces $7 a$-dehydroxylase which catalyzes conversion of primary to secondary bile acids in the colon. Secondary bile acids such as deoxycholic acid (DCA) and lithocholic acid have been known as a promoter of CRC development [51]. DCA from Clostridium and lithocholic acid from Bacteroides fragilis can induce DNA damage, oxidative stress, promote cancer invasion, and activate the mitogen-activated protein kinase (MAPK) pathway and epidermal growth factor receptor signaling [20, 52].

On the other hand, other bile acid, urso-DCA can inhibit tumor genesis by suppressing COX-2 activation
[53]. SCFAs such as butyrate, propionate, and acetate can affect Treg differentiation, induce apoptosis, and interfere cancer genesis. SCFAs are generated by Bifidobacterium, Lactobacillus, and Prevotella from dietary fiber fermentation [20, 54]. Faecalibacterium prausnitzii and Roseburia are some main butyrate-producing bacteria [55]. Butyrate can increase endocan expression which takes role in regulation of inflammation and tumor metastasis through the ERK2/MAPK pathway $[56,57]$. Butyrate also can induce tumor apoptosis through upregulating the Wnt signalling pathway [58].

Some amino acids from undigested diet are used by bacteria for their metabolism and then create metabolic end products; one of them is hydrogen sulfide $\left(\mathrm{H}_{2} \mathrm{~S}\right)[59$, 60]. $\mathrm{H}_{2} \mathrm{~S}$ is produced by sulfate-reducing bacteria from cysteine catabolism [61]. Sulfate-reducing bacteria such as: Fusobacterium, Escherichia, Clostridium, and Strepto- 
coccus metabolize cysteine into $\mathrm{H}_{2} \mathrm{~S}$ and ammonia [62]. Hale et al. [63] provided data that $\mathrm{H}_{2} \mathrm{~S}$ production in CRC samples are more abundant than in noncancerous. An increase $\mathrm{inH}_{2} \mathrm{~S}$ is suspected to be caused by Fusobacteri$u m$ in CRC $[64,65]$. An increase in $\mathrm{H}_{2} \mathrm{~S}$ in the adenomatous colonocytes can favor cell proliferation by stimulating ATP production [66].

EOCRC pathogenesis process and risk exposures are shown in Figure 2. EOCRC is raised in incidence because of westernization of diets and lack of physical activities [50]. A Western diet contains excessive grains, fructose corn syrup, alcohol, fatty meat, and salt. Protein fermentation and bile conjugation are dominant after a person consumes Western diet. These processes can lead to dysbiosis, inflammatory, and carcinogenic state [67]. Excessive salt consumption triggers inflammation by inducing IL-17 and the T-helper cell pathway [68]. These days, children around the world eat more added salt, sugar, fats, and processed food. Deep-frying food generate more proinflammatory advanced glycation end products which play a role in gut dysbiosis. Advanced glycation end products also can be transferred from maternal blood to her child, it increases possibility of EOCRC [69].

CRC in second stage until fourth decade of life is due to prolonged exposure decades earlier [70]. Obesity and excessive body fat are believed to be a cause of inflammation and microbiomes dysbiosis [71]. Physical and social stress in young adults also affect CRC development through microbiomes dysbiosis [50]. Aging process can impact recognition of stimuli and ability to differentiate between self and nonself. Improper reactions to resident microbiota lead to pathogenic processes related to aging [72].

Prolonged exposure of antibiotics also triggers pro-inflammatory and pro-carcinogenicity in colon microbiota. Nitrates and food colorings from drinking or processed meat have been known to be associated with CRC [50]. A systematic review and meta-analysis from Zhao et al. [73] found the association between consumption of red and processed meat with CRC. When cooked at high temperature for a long time, they can produce $\mathrm{N}$-nitroso compound, aromatic hydrocarbons, and heterocyclic amines which may contribute to colorectal carcinogenesis. An increased consumption of red meat $100 \mathrm{~g} /$ day and processed meat $50 \mathrm{~g} /$ day contribute to $20 \%$ risk of CRC. Intake of fiber $10 \mathrm{~g} /$ day gives $10 \%$ reduction of CRC [68]. High-fructose corn syrup, a sugar alternative from starch, leads to obesity, decrease in insulin sensitivity, and gut dysbiosis. In most cases, children are exposed to higher doses of high-fructose corn syrup than adults [50].

Gut Microbiota Profiles in Early- and Late-Onset CRC
Gut microbiome health is reported, it can be affected by early-life exposures (such as maternal infection, nutrition, mode of delivery, breastfeeding, and ethnicity) and specific exposures (such as red meats, western diet, monosodium glutamate, and synthetic dyes) $[29,50]$. High-fat diets cause the accumulation of lipopolysaccharides from bacteria which cause gut inflammation leading to cancer. Dietary emulsifiers can modify gut bacteria and cause colitis, metabolic syndrome, and EOCRC [50]. Early-life exposure like maternal obesity affects gut microbiota composition through transmission of microbiota and metabolites from pregnancy, birth delivery, and breastfeeding [70].

EOCRC patients are more likely to have hereditary CRC syndrome than LOCRC. EOCRC are not usually correlated with typical CRC risk factors, so there are differences in gut microbiome composition with typical LOCRC. Gut microbiota is stabilized after 2 years old and dominated by Bifidobacteria. After that, the variety and diversity of gut microbiota are increased in adults and dominated by Bacteroidetes and Firmicutes; about $90 \%$ of the ecosystem $[20,29]$.

\section{Microbial Profiles in CRC}

Colonic microbiota is mostly consisted of bacteria and can be studied by stool or a biopsy sample. Quantitative PCR, 16S rRNA gene next-generation sequencing, and whole-genome shotgun are the most widely known methods for describing the microbiota community and comparing healthy individual with CRC patients [13]. The 16S rRNA contains 1,500 base pairs which are used for classification of microbiota taxonomy [20]. Nucleic acid samples are read and then compared to the database. Strain, species, genus, and other taxonomic levels can be defined after the analysis [1].

There are many parameters that influence the microbial ecosystem such as age, diet patterns, lifestyle, geographical location, cancer location, and stages. Kim et al. showed that Bacteroidetes, Fusobacteria, and Proteobacteria are increased, while Firmicutes is decreased in CRC patients [74]. Escherichia coli, Enterococcus faecalis, Bacteroides fragilis, and Fusobacterium nucleatum are increased in CRC patients, while Clostridium, Roseburia, and Bifidobacterium are decreased [1]. Figure 3 summarizes the microbial profiles in CRC.

There are also differences between gut microbiota profiles in right- and left-sided CRC. Prevotella and Selenomonas are increased in right CRC, while Fuso- 
Fig. 3. Microbial profiles in CRC. Some bacteria are increased in CRC patients, while others are decreased. Researchers study the oncogenic or anti-oncogenic properties of these bacteria in CRC $[13,20$, 29]. CRC, colorectal cancer.

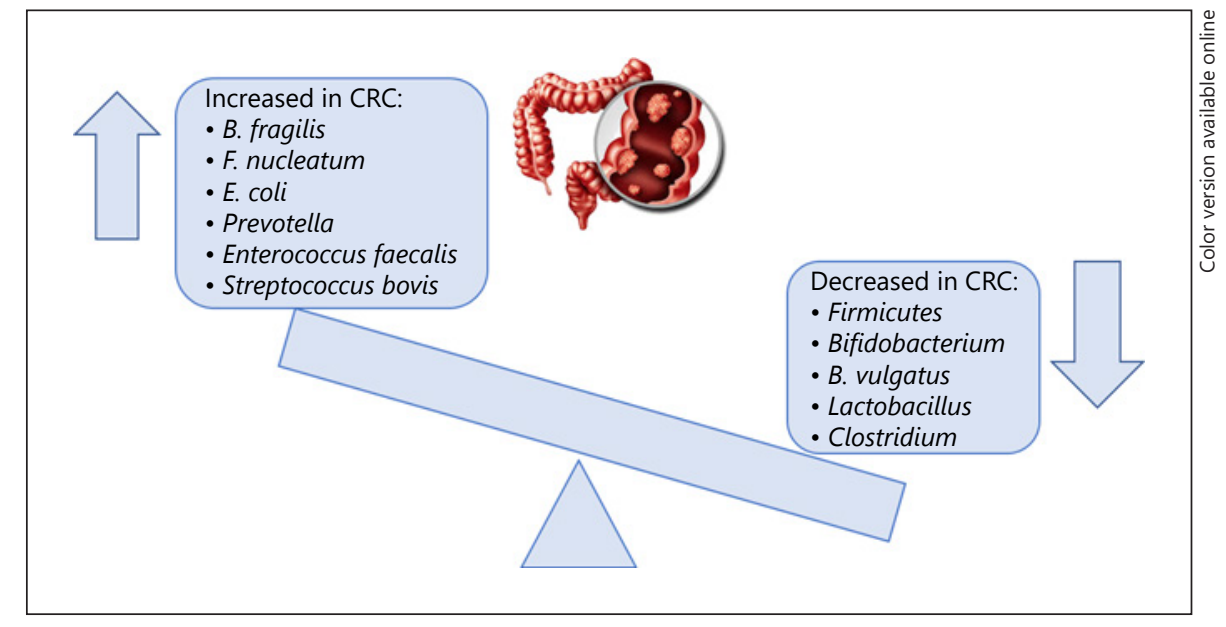

bacterium, Escherichia, and Shigella are increased in left CRC [74]. Gao et al. [75] found that Faecalibacterium and Ruminococcaceae were less abundant in ascending CRC, while Pseudomonas, Lactobacillus, and Streptococcus were more abundant in descending CRC. Toxic metabolites, proteolysis, and biomasses are more abundant in the descending colon than in the ascending colon [76].

\section{Fusobacterium nucleatum}

Fusobacterium nucleatum, a gram-negative oral resident, has been known to be associated with CRC. Kostic et al. [41] proposed that $F$. nucleatum can induce the NF$\kappa \mathrm{B}$ inflammatory pathway and downregulate the T-cell mediated antitumor mechanism. Round et al. [77] suggested that $F$. nucleatum may present virulence factor FadA on the cell surface then begin the WNT signaling pathway. This study also showed that high F. nucleatum abundance was correlated with higher CRC stage and higher mortality. Daily diet can influence suitable microenvironment which induces $F$. nucleatum development and carcinogenesis [29]. F. nucleatum also can activate the $\beta$-catenin pathway and promote chemotherapy resistance due to autophagy induction [11].

\section{Escherichia coli and Bacteroides fragilis}

Some strains of B2 phylogroup Escherichia coli can induce DNA damage, inflammation, and tumorigenesis by producing genotoxin such as colibactin, cytolethal distending toxins, and cytotoxic necrotizing factor [20, 68, 72]. Some bacteria like Bacteroides fragilis and Enterococcus faecalis also produce enterotoxins to cause inflammation and barrier disruption [1].
B. fragilis, an anaerobe commensal bacterium in gut, has 2 classifications: nontoxigenic $B$. fragilis (NTBF) and ETBF. ETBF, which can encode the $B$. fragilis toxin or fragylisin, is enriched in CRC mucosal tissues [20, 29]. $B$. fragilis toxin can induce $\beta$-catenin, NF- $\kappa \mathrm{B}, \mathrm{WNT}$, and MAPK pathways then further promote CRC development [20]. On the other hand, NTBF 9343 strain can induce protective Treg expansion and production of immunosuppresive IL-10 also interfere procarcinogenic Thelper 17 [77, 78]. These benefits have been ascribed to polysaccharide A: the NTBF 9343 strain capsular component [79].

\section{Streptococcus gallolyticus and Helicobacter pylori}

Streptococcus gallolyticus can create collagen I and IV rich biofilms in colonic mucosa. This bacterium is correlated with increased COX-2, IL-1, and IL-8, explaining the chronic inflammatory tumor pathogenesis [29]. Kumar et al. [80] in 2017 studied that S. gallolyticus promoted cell proliferation and CRC development by increasing $\beta$-catenin signaling. A cohort study from Taiwan's National Health Insurance Research Database found that Helicobacter pylori infection was associated with increased risk of CRC (HR 1.87; 95\% CI 1.37-2.57) [81]. Hypergastrinemia from chronic $H$. pylori infection leads to a mutagenesis. Production of VacA and CagA can promote COX-2 and prostaglandin E2 activity then cause chronic inflammation $[29,81]$.

\section{Lactic Acid Bacteria}

Some bacteria also can have a role as a CRC protective. Lactic acid bacteria such as Lactobacillus, Bifidobacterium, and Enterococcus have been known as probiotics 
which can prevent and treat colonic inflammatory diseases and CRC. Intestinal flora indicator usually uses $B i$ fidobacterium to Escherichia (B/E) ratio. Bifidobacterium will decrease and Escherichia will increase in CRC patients. Lactobacillus rhamnosus can take part as an antiinflammatory by reducing the $\beta$-catenin and NF- $\mathrm{BB}$ production [20].

\section{Gut Microbiota Profiles in EOCRC and LOCRC}

Data about microbial profiles in EOCRC are still rare although many researchers have found the correlation between gut microbiota and CRC. The opinion about the role of microbiota dysbiosis and the difference of microbial profiles in younger and older CRC patients are still being discussed. The development of EOCRC may be affected by gut microbiota either dependently or independently (combination with other risk factors) [29].

Weinberg et al. [11] investigated 18 younger (median 39.2 years old) and 13 older (median 72.8 years old) CRC patients in an ongoing study. F. nucleatum was identified in 5 younger patients $(28 \%)$ and 3 older patients $(23 \%)$. There was a much higher presence of $F$. nucleatum in younger CRC patients than previously thought. Moraxella osloensis showed a more significant difference in younger and older CRC patients ( 11 and $46 \% ; p=0.043$, Fisher's exact test). M. osloensis, an anaerobic bacterium from healthy respiratory tract, is a rare pathogen found in immunocompromised patients such as cancer and organ recipients [82, 83]. Some cases of $M$. osloensis infection in cancer include leukemia, melanoma, Hodgkin disease, breast, pancreas, and lung carcinoma $[83,84]$. M. osloensis is difficult to be identified because of similar phenotypic characteristics with other bacteria [85]. This study is still recruiting patients for larger sample sizes so that they can inform better about microbial differences between EOCRC and LOCRC or whether an increase in $F$. nucleatum has a role in the increase of EOCRC [11]. A study in Seoul by Oh et al. [86] revealed that among 593 stage II/III CRC tissues, there was a higher percentage of F. nucleatum in 59 years old or older patients.

Other studies showed that F. nucleatum amount and patient age are not remarkably correlated [29]. A 2017 study by Yamaoka et al. [87] reported that the mean ages of subjects having high $F$. nucleatum abundance and those with low $F$. nucleatum were $67.0 \pm 10.0$ and $66.1 \pm$ 12.8 years, respectively $(p=0.697)$. Mima et al. [88] in 2015 stated that there were not any significant differences of the amount of F. nucleatum with age-groups among

Gut Microbiota Profiles in Early- and Late-Onset CRC
598 CRC cases $(p=0.89)$. Another report by Mima et al. [89] also found no significant difference in F. nucleatum amount and age $(p=0.21$; mean age $=69.3 \pm 8.8)$.

Abdulamir et al. [90] found that age of CRC patients was not associated with previous Streptococcus gallolyticus bacteremia $(p=0.41)$. The mean ages of controls, CRC without bacteremia, and CRC with bacteremia were $57.4 \pm 4.7$, $59.22 \pm 8.18$, and $56.6 \pm 6.7$, respectively. A study from Arafat among Egyptians and Kenyan CRC patients showed a lower amount of Mitsuokella multacida in older CRC patients ( $\geq 50$ years) compared to healthy controls. M. multacida was absent in younger CRC patients ( $<50$ years) $(p=$ 0.01 , compared with older patients). $M$. multacida can produce SCFAs so that it might explain the younger-onset CRC because of the absence of this bacterium [91].

Keshinro et al. [12] with stage I-III microsatellite stable colon cancer specimens from 2014 to 2019 microbial study concluded that there were not any significant differences in the microbial diversity and abundance between EOCRC and LOCRC. They proposed that EOCRC pathogenesis might not be majorly driven by intestinal dysbiosis.

A large meta-analysis from 2,500 individuals (aged 20-89 years) with various diseases such as: CRC, polyps, IBD, diabetes, and liver cirrhosis was carried out to understand gut microbial profiles between age-groups. Ghosh et al. [92] classified age-groups into "young" (2039 years old), "middle-age" (40-59 years old), and "elderly" ( $\geq 60$ years old). They found microbiome differences between the groups and suggested that younger people tended to gain more diseases in microbial taxa (including F. nucleatum, E. coli, B. fragilis, and Streptococci in CRC), while the elderly people tended to lose usually found microbes in the healthy gut.

Based on those studies we can assume that microbiota does vary with age, and EOCRC may be associated with some obvious bacteria. Further researches are required to understand why microbiomes change with age and what are the outcomes of these changes for diseases development (including CRC) based on age-groups. Investigating gut microbiota profiles in EOCRC patients may provide new insights in diagnostic biomarkers or even further therapy in the future.

\section{Conclusion}

Last decade trends showed a persistent rise of EOCRC incidence and mortality, primarily involving the distal colon and rectum. Microbiota have a role in carcinogen- 
esis through different ways, particularly in dysbiosis condition. Many studies point out microbial differences between CRC and healthy patients. Each bacterium has a specific role in promoting chronic inflammation or immunity dysregulation which then leads to cancer genesis. Some researchers also show data about gut microbiota profiles in EOCRC and LOCRC, but the differences have not been significant yet. Larger samples and further studies are needed to give a better view on these differences, and whether gut dysbiosis itself is responsible for EOCRC genesis. Gut microbiota profiling in EOCRC and LOCRC may give promising explanations about increasing incidence of EOCRC and may be useful for further cancer diagnostic marker or therapeutic objectives.

\section{Conflict of Interest Statement}

The authors have no conflicts of interest to declare.

\section{Funding Sources}

This study was supported by Hibah Kolaborasi Riset Internasional University of Indonesia 2019 (NKB-1929/UN2.R3.1/ HKP.05.00/2019), which was budgeted through Directorate of Research and Community Engagement, University of Indonesia.

\section{Author Contributions}

Murdani Abdullah designed and conceptualized the structure of the manuscript; Ninik Sukartini and Saskia Aziza Nursyirwan critically reviewed the manuscript; Rabbinu Rangga Pribadi, Hasan Maulahela, Amanda Pitarini Utari, and Virly Nanda Muzellina provided the literatures search and review; Agustinus Wiraatmadja developed the manuscript and combined the provided literatures; and Kaka Renaldi arranged the figures and references.

\section{References}

1 Saus E, Iraola-Guzmán S, Willis JR, BrunetVega A, Gabaldón T. Microbiome and colorectal cancer: roles in carcinogenesis and clinical potential. Mol Aspects Med. 2019;69: 93-106.

2 Arnold M, Sierra MS, Laversanne M, Soerjomataram I, Jemal A, Bray F. Global patterns and trends in colorectal cancer incidence and mortality. Gut. 2017;66(4):683-91.

3 Moskal A, Freisling H, Byrnes G, Assi N, Fahey MT, Jenab M, et al. Main nutrient patterns and colorectal cancer risk in the European Prospective Investigation into Cancer and Nutrition study. Br J Cancer. 2016; 115(11):1430-40

4 You YN, Lee LD, Deschner BW, Shibata D. Colorectal cancer in the adolescent and young adult population. JCO Oncol Pract. 2020; 16(1):19-27.

5 Chen FW, Sundaram V, Chew TA, Ladabaum $\mathrm{U}$. Low prevalence of criteria for early screening in young-onset colorectal cancer. Am J Prev Med. 2017;53(6):933.

6 Wolf AM, Fontham ET, Church TR, Flowers CR, Guerra CE, LaMonte SJ, et al. Colorectal cancer screening for average-risk adults: 2018 guideline update from the American Cancer Society. CA Cancer J Clin. 2018;68(4):250-81.

7 Campos FG. Colorectal cancer in young adults: a difficult challenge. World J Gastroenterol. 2017;23(28):5041.
8 Silla IO, Rueda D, Rodríguez Y, García JL, de la Cruz Vigo F, Perea J. Early-onset colorectal cancer: a separate subset of colorectal cancer. World J Gastroenterol. 2014;20(46): 17288.

9 Gausman V, Dornblaser D, Anand S, Hayes $\mathrm{RB}, \mathrm{O}$ 'Connell K, Du M, et al. Risk factors associated with early-onset colorectal cancer. Clin Gastroenterol Hepatol. 2020;18(12): 2752-9.e2.

10 Lee LD, You YN, editors. Young-onset colorectal cancer: diagnosis and management. Sem Colon Rectal Surg. 2018;29(3):98101.

11 Weinberg BA, Wang H, Geng X, Shokralla S, Bakhshi E, Chaldekas K, et al. A comparison study of the intratumoral microbiome in younger verses older-onset colorectal cancer (COSMO CRC). ASCO 2020 GI Cancers Symposium on January 25, 2020, San Francisco, California; 2020.

12 Keshinro A, Vanderbilt C, Stadler ZK, Shia J, Gonen M, Chen C-T, et al. Do differences in the microbiome explain early onset in colon cancer? J Clin Oncol. 2020;38(15).

13 Villéger R, Lopès A, Veziant J, Gagnière J, Barnich N, Billard E, et al. Microbial markers in colorectal cancer detection and/or prognosis. World J Gastroenterol. 2018; 24(22):2327.

14 Leuraud K, Jezewski-Serra D, Viguier J, Salines E. Colorectal cancer screening by guaiac faecal occult blood test in France: evaluation of the programme two years after launching. Cancer Epidemiol. 2013;37(6):959-67.
15 Bevan R, Rutter MD. Colorectal cancer screening-who, how, and when? Clin Endosc. 2018;51(1):37.

16 Yu LC, Wei SC, Ni YH. Impact of microbiota in colorectal carcinogenesis: lessons from experimental models. Intest Res. 2018;16(3): 346.

17 Flemer B, Warren RD, Barrett MP, Cisek K, Das A, Jeffery IB, et al. The oral microbiota in colorectal cancer is distinctive and predictive. Gut. 2018;67(8):1454-63.

18 Triantafillidis JK, Vagianos C, Malgarinos G. Colonoscopy in colorectal cancer screening: current aspects. Indian J Surg Oncol. 2015; 6(3):237-50.

19 McLachlan SA, Clements A, Austoker J. Patients' experiences and reported barriers to colonoscopy in the screening context: a systematic review of the literature. Patient Educ Couns. 2012;86(2):137-46.

20 Zou S, Fang L, Lee MH. Dysbiosis of gut microbiota in promoting the development of colorectal cancer. Gastroenterol Rep. 2018; 6(1):1-12.

21 Sears CL, Garrett WS. Microbes, microbiota, and colon cancer. Cell Host Microbe. 2014; 15(3):317-28.

22 Gehart H, Clevers H. Tales from the crypt: new insights into intestinal stem cells. Nat Rev Gastroenterol Hepatol. 2019;16(1):19-34.

23 Flint HJ, Scott KP, Duncan SH, Louis P, Forano E. Microbial degradation of complex carbohydrates in the gut. Gut Microbes. 2012; 3(4):289-306. 
24 Yoo JY, Groer M, Dutra SVO, Sarkar A, McSkimming DI. Gut microbiota and immune system interactions. Microorganisms. 2020; 8(10): 1587.

25 Zhou L, Sonnenberg GF. Essential immunologic orchestrators of intestinal homeostasis. Sci Immunol. 2018;3(20):eaao1605.

26 Janney A, Powrie F, Mann EH. Host-microbiota maladaptation in colorectal cancer. $\mathrm{Na}$ ture. 2020;585(7826):509-17.

27 Karin M, Clevers H. Reparative inflammation takes charge of tissue regeneration. Nature. 2016;529(7586):307-15.

28 Lamichane S, Dahal Lamichane B, Kwon SM. Pivotal roles of peroxisome proliferator-activated receptors (PPARs) and their signal cascade for cellular and whole-body energy homeostasis. Int J Mol Sci. 2018;19(4):949.

29 Mukherji R, Weinberg BA. The gut microbiome and potential implications for early-onset colorectal cancer. Colorectal Cancer. 2020; 9(3):CRC25.

30 Savari S, Vinnakota K, Zhang Y, Sjölander A Cysteinyl leukotrienes and their receptors: bridging inflammation and colorectal cancer. World J Gastroenterol. 2014;20(4):968.

31 Loddo I, Romano C. Inflammatory bowel disease: genetics, epigenetics, and pathogenesis. Front Immunol. 2015;6:551.

32 Zheng D, Liwinski T, Elinav E. Interaction between microbiota and immunity in health and disease. Cell Res. 2020;30:492-506.

33 Fulde M, Sommer F, Chassaing B, van Vorst K, Dupont A, Hensel M, et al. Neonatal selection by Toll-like receptor 5 influences longterm gut microbiota composition. Nature. 2018;560(7719):489-93.

34 Tjalsma H, Boleij A, Marchesi JR, Dutilh BE A bacterial driver-passenger model for colorectal cancer: beyond the usual suspects. Nat Rev Microbiol. 2012;10(8):575-82.

35 Prorok-Hamon M, Friswell MK, Alswied A, Roberts CL, Song F, Flanagan PK, et al. Colonic mucosa-associated diffusely adherent afaC+ Escherichia coli expressing lpfA and pks are increased in inflammatory bowel disease and colon cancer. Gut. 2014;63(5):761-70.

36 Bonnet M, Buc E, Sauvanet P, Darcha C, Dubois D, Pereira B, et al. Colonization of the human gut by E. coli and colorectal cancer risk. Clin Cancer Res. 2014;20(4):859-67.

37 Viljoen KS, Dakshinamurthy A, Goldberg P, Blackburn JM. Quantitative profiling of colorectal cancer-associated bacteria reveals associations between fusobacterium spp., enterotoxigenic Bacteroides fragilis (ETBF) and clinicopathological features of colorectal cancer. PloS One. 2015;10(3):e0119462.

38 Fearon ER. Molecular genetics of colorectal cancer. Annu Rev Pathol. 2011;6:479-507.

39 Raskov H, Burcharth J, Pommergaard HC. Linking gut microbiota to colorectal cancer. J Cancer. 2017;8(17):3378.

40 Marchesi JR, Dutilh BE, Hall N, Peters WH, Roelofs R, Boleij A, et al. Towards the human colorectal cancer microbiome. PLoS One. 2011;6(5):e20447.
41 Kostic AD, Chun E, Robertson L, Glickman JN, Gallini CA, Michaud M, et al. Fusobacterium nucleatum potentiates intestinal tumorigenesis and modulates the tumor-immune microenvironment. Cell Host Microbe. 2013; 14(2):207-15

42 Bedford A, Gong J. Implications of butyrate and its derivatives for gut health and animal production. Anim Nutr. 2018;4(2):151-9.

43 Hajishengallis G, Darveau RP, Curtis MA. The keystone-pathogen hypothesis. Nat Rev Microbiol. 2012;10(10):717-25.

44 Geng J, Song Q, Tang X, Liang X, Fan H, Peng $\mathrm{H}$, et al. Co-occurrence of driver and passenger bacteria in human colorectal cancer. Gut Pathog. 2014;6(1):26.

45 Loeb LA, Harris CC. Advances in chemical carcinogenesis: a historical review and prospective. Cancer Res. 2008;68(17):6863-72.

46 Lopez LR, Bleich RM, Arthur JC. Microbiota effects on carcinogenesis: initiation, promotion and progression. Annu Rev Med. 2020; 72:243-61.

47 Cuevas-Ramos G, Petit CR, Marcq I, Boury M, Oswald E, Nougayrède JP. Escherichia coli induces DNA damage in vivo and triggers genomic instability in mammalian cells. Proc Natl Acad Sci U S A. 2010;107(25):11537-42.

48 Hurtado CG, Wan F, Housseau F, Sears CL. Roles for interleukin 17 and adaptive immunity in pathogenesis of colorectal cancer. Gastroenterology. 2018;155(6):1706-15.

49 Rubinstein MR, Wang X, Liu W, Hao Y, Cai G, Han YW. Fusobacterium nucleatum promotes colorectal carcinogenesis by modulating $\mathrm{E}$-cadherin $/ \beta$-catenin signaling via its FadA adhesin. Cell Host Microbe. 2013;14(2): 195-206.

50 Hofseth LJ, Hebert JR, Chanda A, Chen H, Love BL, Pena MM, et al. Early-onset colorectal cancer: initial clues and current views. Nat Rev Gastroenterol Hepatol. 2020;17(6):352-64.

51 Ridlon JM, Kang DJ, Hylemon PB, Bajaj JS. Bile acids and the gut microbiome. Curr Opin Gastroenterol. 2014;30(3):332.

52 Baek MK, Park JS, Park JH, Kim MH, Kim $\mathrm{HD}$, Bae WK, et al. Lithocholic acid upregulates $\mathrm{UPAR}$ and cell invasiveness via MAPK and AP-1 signaling in colon cancer cells. Cancer Lett. 2010;290(1):123-8

53 Centuori SM, Martinez JD. Differential regulation of EGFR-MAPK signaling by deoxycholic acid (DCA) and ursodeoxycholic acid (UDCA) in colon cancer. Dig Dis Sci. 2014; 59(10):2367-80.

54 Hester CM, Jala VR, Langille MG, Umar S, Greiner KA, Haribabu B. Fecal microbes, short chain fatty acids, and colorectal cancer across racial/ethnic groups. World J Gastroenterol. 2015;21(9):2759.

55 Miquel S, Martín R, Rossi O, Bermúdez-Humarán LG, Chatel JM, Sokol H, et al. Faecalibacterium prausnitzii and human intestinal health. Curr Opin Microbiol. 2013;16(3): 255-61.
56 Zuo L, Zhang SM, Hu RL, Zhu HQ, Zhou Q, Gui SY, et al. Correlation between expression and differentiation of endocan in colorectal cancer. World J Gastroenterol. 2008;14(28): 4562.

57 Zuo L, Lu M, Zhou Q, Wei W, Wang Y. Butyrate suppresses proliferation and migration of RKO colon cancer cells though regulating endocan expression by MAPK signaling pathway. Food Chem Toxicol. 2013;62:892-900.

58 Lazarova DL, Bordonaro M, Carbone R, Sartorelli AC. Linear relationship between Wnt activity levels and apoptosis in colorectal carcinoma cells exposed to butyrate. Int J Cancer. 2004;110(4):523-31.

59 Davila AM, Blachier F, Gotteland M, Andriamihaja M, Benetti PH, Sanz Y, et al. Intestinal luminal nitrogen metabolism: role of the gut microbiota and consequences for the host. Pharmacol Res. 2013;68(1):95-107.

60 Scott KP, Gratz SW, Sheridan PO, Flint HJ, Duncan SH. The influence of diet on the gut microbiota. Pharmacol Res. 2013;69(1):5260.

61 Guo FF, Yu TC, Hong J, Fang JY. Emerging roles of hydrogen sulfide in inflammatory and neoplastic colonic diseases. Front Physiol. 2016;7:156.

62 Barton LL, Ritz NL, Fauque GD, Lin HC. Sulfur cycling and the intestinal microbiome. Dig Dis Sci. 2017;62(9):2241-57.

63 Hale VL, Jeraldo P, Mundy M, Yao J, Keeney $\mathrm{G}$, Scott N, et al. Synthesis of multi-omic data and community metabolic models reveals insights into the role of hydrogen sulfide in colon cancer. Methods. 2018;149:59-68.

64 Rubinstein MR, Baik JE, Lagana SM, Han RP, Raab WJ, Sahoo D, et al. Fusobacterium nucleatum promotes colorectal cancer by inducing $\mathrm{Wnt} / \beta$-catenin modulator Annexin $\mathrm{A} 1$. EMBO Rep. 2019;20(4):e47638

65 Bullman S, Pedamallu CS, Sicinska E, Clancy TE, Zhang X, Cai D, et al. Analysis of Fusobacterium persistence and antibiotic response in colorectal cancer. Science. 2017;358(6369): 1443-8.

66 Blachier F, Andriamihaja M, Larraufie P, Ahn E, Lan A, Kim E. Production of hydrogen sulfide by the intestinal microbiota and epithelial cells and consequences for the colonic and rectal mucosa. Am J Physiol Gastrointest Liver Physiol. 2021;320(2):G125-35.

67 Ohgaki H, Kusama K, Matsukura N, Morino K, Hasegawa H, Sato S, et al. Carcinogenicity in mice of a mutagenic compound, 2-amino3-methylimidazo[4,5-f]quinoline, from broiled sardine, cooked beef and beef extract. Carcinogenesis. 1984;5(7):921-4.

68 Niederreiter L, Adolph TE, Tilg H. Food, microbiome and colorectal cancer. Dig Liver Dis. 2018;50(7):647-52.

69 Uribarri J, Woodruff S, Goodman S, Cai W, Chen X, Pyzik R, et al. Advanced glycation end products in foods and a practical guide to their reduction in the diet. J Am Diet Assoc. 2010;110(6):911-16.e12. 
70 Zhang Q, Berger FG, Love B, Banister CE, Murphy EA, Hofseth LJ. Maternal stress and early-onset colorectal cancer. Med Hypotheses. 2018;121:152-9.

71 Siegel RL, Fedewa SA, Anderson WF, Miller $\mathrm{KD}, \mathrm{Ma}$ J, Rosenberg PS, et al. Colorectal cancer incidence patterns in the United States, 1974-2013. J Natl Cancer Inst. 2017;109(8): djw322.

72 Tibbs TN, Lopez LR, Arthur JC. The influence of the microbiota on immune development, chronic inflammation, and cancer in the context of aging. Microb Cell. 2019;6(8):324.

73 Zhao Z, Feng Q, Yin Z, Shuang J, Bai B, Yu P, et al. Red and processed meat consumption and colorectal cancer risk: a systematic review and meta-analysis. Oncotarget. 2017;8(47):83306.

74 Kim K, Castro EJT, Shim H, Advincula JVG, Kim YW. Differences regarding the molecular features and gut microbiota between right and left colon cancer. Ann Coloproctol. 2018; 34(6):280.

75 Gao R, Kong C, Huang L, Li H, Qu X, Liu Z et al. Mucosa-associated microbiota signature in colorectal cancer. Eur J Clin Microbiol Infect Dis. 2017;36(11):2073-83.

76 Alhinai EA, Walton GE, Commane DM. The role of the gut microbiota in colorectal cancer causation. Int J Mol Sci. 2019;20(21):5295.

77 Round JL, Lee SM, Li J, Tran G, Jabri B, Chatila TA, et al. The Toll-like receptor 2 pathway establishes colonization by a commensal of the human microbiota. Science. 2011; 332(6032):974-7.

78 Round JL, Mazmanian SK. Inducible Foxp3+ regulatory T-cell development by a commen- sal bacterium of the intestinal microbiota. Proc Natl Acad Sci U S A. 2010;107(27): 12204-9.

79 Chan JL, Wu S, Geis AL, Chan GV, Gomes TAM, Beck SE, et al. Non-toxigenic Bacteroides fragilis (NTBF) administration reduces bacteria-driven chronic colitis and tumor development independent of polysaccharide A. Mucosal Immunol. 2019;12(1):164-77.

80 Kumar R, Herold JL, Schady D, Davis J, Kopetz S, Martinez-Moczygemba M, et al. Streptococcus gallolyticus subsp. gallolyticus promotes colorectal tumor development. PLoS Pathog. 2017;13(7):e1006440.

81 Liu IL, Tsai CH, Hsu CH, Hu JM, Chen YC, Tian YF, et al. Helicobacter pylori infection and the risk of colorectal cancer: a nationwide population-based cohort study. QJM. 2019; 112(10):787-92.

82 Maruyama Y, Shigemura T, Aoyama K, Nagano N, Nakazawa Y. Bacteremia due to Moraxella osloensis: a case report and literature review. Braz J Infect Dis. 2018;22(1):60-2.

83 Han XY, Tarrand JJ. Moraxella osloensis blood and catheter infections during anticancer chemotherapy: clinical and microbiologic studies of 10 cases. Am J Clin Pathol. 2004, 121(4):581-7.

84 Denham JD, Nanjappa S, Greene JN. Moraxella species bacteremia in cancer patients: a case series and review of the literature. Infect Dis Clin Pract. 2018;26(4):188-90.

85 Roh KH, Kim CK, Koh E, Kim MS, Yong D, Park SC, et al. Three cases of Moraxella osloensis meningitis: a difficult experience in species identification and determination of clinical significance. J Korean Med Sci. 2010; 25(3):501.

86 Oh HJ, Kim JH, Bae JM, Kim HJ, Cho NY, Kang GH. Prognostic impact of Fusobacterium nucleatum depends on combined tumor location and microsatellite instability status in stage II/III colorectal cancers treated with adjuvant chemotherapy. J Pathol Transl Med. 2019;53(1):40.

87 Yamaoka Y, Suehiro Y, Hashimoto S, Hoshida T, Fujimoto M, Watanabe M, et al. Fusobacterium nucleatum as a prognostic marker of colorectal cancer in a Japanese population. J Gastroenterol. 2018;53(4):517-24.

88 Mima K, Sukawa Y, Nishihara R, Qian ZR, Yamauchi M, Inamura K, et al. Fusobacterium nucleatum and $\mathrm{T}$ cells in colorectal carcinoma. JAMA Oncol. 2015;1(5):653-61.

89 Mima K, Nishihara R, Qian ZR, Cao Y, Sukawa Y, Nowak JA, et al. Fusobacterium nucleatum in colorectal carcinoma tissue and patient prognosis. Gut. 2016;65(12):1973-80.

90 Abdulamir AS, Hafidh RR, Bakar FA. Molecular detection, quantification, and isolation of Streptococcus gallolyticus bacteria colonizing colorectal tumors: inflammation-driven potential of carcinogenesis via IL-1, COX-2, and IL-8. Mol Cancer. 2010;9(1):249.

91 Arafat W. P-316 Profile of microbiota is associated with early onset of colorectal cancer in Egyptian and Kenyan patients. Ann Oncol. 2020;31:S192.

92 Ghosh TS, Das M, Jeffery IB, O’Toole PW. Adjusting for age improves identification of gut microbiome alterations in multiple diseases. Elife. 2020;9:e50240. 